EFI-96-04

hep-th/9602049

\title{
New Principles for String/Membrane Unification
}

\author{
David Kutasov and Emil Martinec* \\ Enrico Fermi Inst. and Dept. of Physics \\ University of Chicago \\ 5640 S. Ellis Ave., Chicago, IL 60637
}

\begin{abstract}
The target space theory of the $\mathrm{N}=(2,1)$ heterotic string may be interpreted as a theory of gravity coupled to matter in either $1+1$ or $2+1$ dimensions. Among the target space theories in $1+1$ dimensions are the bosonic, type II, and heterotic string world sheet field theories in a physical gauge. The $2+1$ dimensional version describes a consistent quantum theory of supermembranes in $10+1$ dimensions. The unifying framework for all of these vacua is a theory of $2+2$ dimensional self-dual geometries embedded in $10+2$ dimensions. There are also indications that the $\mathrm{N}=(2,1)$ string describes the strong coupling dynamics of compactifications of critical string theories to two dimensions, and may lead to insights about the fundamental degrees of freedom of the theory.
\end{abstract}

$2 / 96$

* Supported in part by Dept. of Energy grant DEFG02-90ER-40560. 


\section{Introduction}

Recent progress in string duality (for a recent status report, see [1]) indicates that there is but one unified theory whose low-energy manifestations include all string theories, as well as eleven-dimensional supergravity. This poses a puzzle, since the latter seems to be a theory of quantum membranes (sometimes called M-theory), whereas the usual worldvolume analysis of such theories [2] as 2+1 (super-) gravity coupled to matter does not lead to a renormalizable quantum theory. Moreover, the classical equations of motion are highly nonlinear and difficult to solve, hardly a promising starting point. Trying to turn these shortcomings into virtues, some have speculated that, since the eleven-dimensional theory has no small dimensionless coupling, there ought not to be a sensible theory of 'free' quantum membranes. However, deeper understanding ought to arise if we can find new principles by which to construct the quantum theory of membranes, hopefully in tandem with a new approach to string theory that incorporates all of its various manifestations. We believe that $\mathrm{N}=(2,1)$ heterotic string theory shows us the way to such new principles.

In this paper, we are going to revive an old idea of Green's [3]: That string theories whose target space dynamics is effectively two-dimensional have a low-energy effective action which is the worldsheet field theory of another string. This is all but guaranteed by the fact that the target space of string theory always contains gravity, and two-dimensional gravity coupled to matter is by definition string theory. What is not guaranteed is that one obtains critical string theory in this way; in fact, most of Green's models were noncritical strings. As one increases the worldsheet gauge symmetry of string theory, one comes closer to realizing critical strings in the two-dimensional target space. It turns out that $\mathrm{N}=(2,1)$ local supersymmetry is the magical principle which generates all critical string theories, as well as membranes. For lower supersymmetry, one generally has (a) infinite towers of massive states, leading to complicated effective interactions for the massless modes; and (b) the wrong number of massless fields. $\mathrm{N}=(2,2)$ supersymmetry seems too restrictive.

$\mathrm{N}=2$ strings have been studied by many authors over the years (for a review, see [4]; for early work, see [5, 3 ). The $\mathrm{N}=2$ string properly lives in two complex target dimensions, with $4+0$ or $2+2$ signature. Ooguri and Vafa [6-8] systematically investigated the geometry for these theories. This geometry is self-dual, admitting a twistorial formulation [7,9]. Thus the $\mathrm{N}=(2,2)$ closed and open string theories describe some sort of quantization of self-dual gravity and self-dual Yang-Mills, respectively. The heterotic $\mathrm{N}=(2,1)$ and $(2,0)$ theories add a new wrinkle. The right-moving worldsheet gauge invariance requires $2+2$ 
signature, which would spoil the no-ghost theorem for the left-moving tower of modes. Ooguri and Vafa therefore add two target dimensions to the left-movers, such that the left movers are in $10+2$ or $26+2$ dimensions; and simultaneously enlarge the left-moving gauge principle to include a null current algebra, to factor out the unwanted additional timelike modes. Any null current will do; if we choose its orientation entirely within the subspace common to both left- and right-movers, the effective target space dynamics is reduced from $2+2$ to $1+1$ dimensions. Thus heterotic $\mathrm{N}=2$ strings actually do describe the $1+1$ dimensional target space kinematics employed in [3]. In addition, Ooguri and Vafa point out another possibility: One may orient the null current partly in the $2+2$ spacetime and partly in the 'internal space' of the left-movers. This effectively removes only one (timelike) direction from the $2+2$ target, leaving a $2+1$ dimensional target space dynamics. This theory contains a kind of gravity inherited from self-dual gravity in four dimensions, together with matter fields depending on the details of the construction.

The version of the $(2,1)$ heterotic string with $1+1$ dimensional target has several vacua, depending on a choice of GSO projection. Below we will exhibit examples having (a) 24 bosons; (b) 8 bosons, 8 left-moving, and 8 right-moving fermions with $(8,8)$ supersymmetry; and (c) 8 bosons, 8 right-moving fermions, and 16 chiral left-moving bosons with $(8,0)$ supersymmetry. These target space theories are free and have no massive excitations; they are, respectively, the bosonic, type II, and heterotic string world sheet field theories in a physical gauge. The construction of the target space heterotic string involves an orbifold that can only be seen from the $2+2$ dimensional point of view.

The $(2,1)$ heterotic string with $2+1$ dimensional target space has a version containing 8 bosons, 8 fermions, having $\mathrm{N}=8$ supersymmetry; and provides a promising candidate for a consistent quantum theory of supermembranes in 11 dimensions. Two features of this theory stand out: First, the classical theory is integrable, being a restricted form of $2+2$ self-dual gravity coupled to self-dual matter; second, the $(2,1)$ string gives us some prescription for the quantum theory (it is not ruled out that one might be able to quantize directly the target space effective field theory).

Our plan is as follows: In section 2, we briefly review the $\mathrm{N}=(2,1)$ heterotic string and establish our conventions. In section 3 we consider models whose dynamics appears $1+1$ dimensional in the $2+2$ target space by choosing the null current orthogonal to the left-moving internal space. We exhibit choices of GSO projection which yield bosonic, type II, and heterotic strings as the effective target space field theories. In section 4, we turn to the models which appear $2+1$ dimensional, with null current partly in the internal 
space. A particular choice of GSO projection gives a candidate for the membrane of eleven-dimensional supergravity; another gives a bosonic membrane in 27 dimensions. All discussion in these two sections will take place in the context of flat target geometries. In section 5, we briefly examine the sigma model describing the curved space generalization of the theory. We conclude in section 6 with a list of comments and speculations.

\section{2. $\mathrm{N}=(2,1)$ Heterotic Strings}

In this paper we will be mostly discussing $(2,1)$ strings, heterotic strings with $N=2$ local supersymmetry for the right movers, and $N=1$ supersymmetry for the left movers. Since many aspects of the construction are common to all the models we will discuss, we will start with a review of some of the relevant features of the right-moving $N=2$ and the left-moving $N=1$ chiral sectors. This will also serve to establish our notation. In the next sections we will discuss different ways of putting the two sectors together. We will be using rather heavily the technology developed in [10]; the reader may want to consult this reference for further details. For more details on $N=2$ strings, see e.g. [5] 8 .

\subsection{The right movers: $N=2$ strings.}

In flat spacetime $\mathbb{R}^{2,2}$, the right movers of the $\mathrm{N}=(2,1)$ heterotic string are four real scalar fields $x^{\mu}, \mu=0,1,2,3$ with signature $(-,-,+,+)$, and their superpartners under the $N=2$ superconformal algebra, $\bar{\psi}^{\mu}$. We will sometimes bosonize the fermions $\bar{\psi}$, as:

$$
\begin{aligned}
& \bar{\psi}^{0} \pm \bar{\psi}^{3}=e^{ \pm i \bar{H}_{1}} \\
& \bar{\psi}^{1} \pm \bar{\psi}^{2}=e^{ \pm i \bar{H}_{2}}
\end{aligned}
$$

and will freely switch between the fermionic and bosonic representations. The $N=2$ superconformal generators can be written as:

$$
\begin{aligned}
\bar{T} & =-\frac{1}{2} \bar{\partial} x \bar{\partial} x-\bar{\psi} \bar{\partial} \bar{\psi} \\
\bar{G}^{ \pm} & =e^{ \pm i \bar{H}_{1}} \bar{\partial}\left(x^{0} \pm x^{3}\right)+e^{ \pm i \bar{H}_{2}} \bar{\partial}\left(x^{1} \pm x^{2}\right) \\
\bar{J} & =i \bar{\partial} \bar{H}_{1}+i \bar{\partial} \bar{H}_{2}
\end{aligned}
$$

To construct physical states in the covariant BRST formalism one also needs the ghosts for the gauge algebra generated by $\bar{T}, \bar{G}_{ \pm}, \bar{J}$; these will be denoted by $(\bar{b}, \bar{c}),\left(\bar{\beta}_{ \pm}, \bar{\gamma}_{ \pm}\right)$, and

$(\overline{\tilde{b}}, \overline{\tilde{c}})$, respectively. In particular vertex operators will turn out to depend on the fields $\bar{\phi}_{ \pm}$ arising from the bosonization of $\bar{\beta}_{ \pm}, \bar{\gamma}_{ \pm}$in the usual way [10: $\bar{\beta}_{ \pm} \bar{\gamma}_{ \pm}=\bar{\partial} \bar{\phi}_{ \pm}$. 
Most (but not all) vertex operators we will need will have the same form as far as the right-moving $N=2$ string part is concerned:

$$
\begin{aligned}
V_{-1}(k) & =e^{-\bar{\phi}_{+}-\bar{\phi}_{-}} e^{i k \cdot x} \\
V_{0}(k) & =\bar{G}_{-\frac{1}{2}}^{+} \bar{G}_{-\frac{1}{2}}^{-} e^{i k \cdot x}
\end{aligned}
$$

with the first line describing the vertex operator in the -1 picture, and the second, in the 0 picture.

Three point functions of physical states reduce to products of right- and left-moving parts; the $N=2$ right-moving part contributes

$$
\left\langle V_{-1}(k) V_{-1}(p) V_{0}(r)\right\rangle=\left(k_{0}+k_{3}\right)\left(p_{0}-p_{3}\right)+\left(k_{1}+k_{2}\right)\left(p_{1}-p_{2}\right)-(k \leftrightarrow p) .
$$

We will use below the fact that when one reduces all the momenta such that $k_{0}+k_{3}=$ $p_{0}+p_{3}=r_{0}+r_{3}=0$, the amplitude (2.4) vanishes.

\subsection{The left-moving $N=1$ sector.}

To describe the left-moving sector we need to find a $\hat{c}=10 N=1$ superconformal field theory (SCFT). The $2+2$ dimensional spacetime coordinates $x^{\mu}$ (which are non-compact and hence shared by the left and right movers), and their superpartners, $\psi^{\mu}$ form a $\hat{c}=4$ SCFT. Naively we need an additional $\hat{c}=6$, but there is a subtlety [8]. As explained in the introduction, ghost elimination requires us to gauge a null $U(1)$ supercurrent on the left side. The ghosts corresponding to this $U(1)$ carry $\hat{c}=-2$ so overall we are looking for an internal $\hat{c}=8(c=12)$ SCFT. A convenient representation of a large class of such theories is provided by twenty-four free fermions, $\lambda^{a}$ [11]. The total $N=1$ superconformal algebra for the left movers is:

$$
\begin{aligned}
& T=-\frac{1}{2} \partial x \partial x-\psi \partial \psi-\lambda \partial \lambda \\
& G=\psi \partial x+\frac{1}{6} f_{a b c} \lambda^{a} \lambda^{b} \lambda^{c} .
\end{aligned}
$$

Here $f_{a b c}$ are the structure constants of some semisimple group of dimension 24, under which the $\lambda^{a}$ transform as the adjoint representation. As for the $N=2$ case, there are ghosts $b, c, \beta, \gamma$ needed to gauge (2.5); as well as ghosts of the supersymmetric $U(1)$ current algebra, which we will suppress. Some vertex operators depend on the bosonized $\beta, \gamma$ ghost $\beta \gamma=\partial \phi$. A special case of (2.5) that we will use is obtained by bosonizing sixteen of the twenty-four fermions, and describing the internal SCFT in terms of eight 
left-moving scalars $y^{a}$ (living on the $E_{8}$ torus for modular invariance) and eight left-moving fermions, $\lambda^{a}$.

As mentioned above, to define a vacuum of the $N=(2,1)$ string one needs to identify in the left-moving $N=1 \mathrm{SCFT}$ a null $U(1)$ supersymmetric current algebra, that is then gauged. There are two fundamentally inequivalent choices:

1) If the $U(1)$ lies entirely in the 'spacetime', $\mathbb{R}^{2,2}$ part of the theory, one finds a theory that effectively is dimensionally reduced from $2+2$ to $1+1$ dimensions. We will use the supercurrent $\left(\psi^{0}+\psi^{3}\right)+\theta\left(\partial x^{0}+\partial x^{3}\right)$, which reduces the target space to $\mathbb{R}^{1,1}$ parametrized by $\left(x^{1}, x^{2}\right)$.

2 ) If the $U(1)$ lies partly in spacetime and partly in the internal theory, $J=\partial x^{0}+$ $J_{\text {internal, }}$, we find a theory in $1+2$ non-compact dimensions, with a second compact time dimension $x^{0}$.

In addition to the choice above, the theories are also parametrized by the choice of GSO projection on the fermions $\lambda^{a}$ etc. In the next sections we will describe the physics of the different theories that arise from various natural choices.

\section{Target Space Strings}

Above we saw that $N=(2,1)$ theories fall into two broad classes, depending on the choice of the null $U(1)$ (actually $\mathbb{R}$ ) super-current algebra that is gauged on the left-moving, $N=1$ side. If the $U(1)$ lies purely in the four dimensional "spacetime" part of the $(2,1)$ sigma model, we find theories whose target space is $1+1$ dimensional. These theories will be discussed in this section. A $U(1)$ that lies partly in spacetime and partly in the internal supeconformal field theory leads to theories in $2+1$ dimensional target space; such theories will be described in the next section. Eventually we will see that, regardless of such a choice of $U(1)$ embedding, the theory is more properly thought of in $2+2$ dimensions.

String theory is a theory of spacetime gravity. Therefore, when the target space of the $(2,1)$ string is two-dimensional, we expect the spacetime dynamics to describe matter coupled to two-dimensional gravity. We will in fact see that the two-dimensional gravity systems that arise correspond to various models of critical strings, with the two-dimensional target space of the $(2,1)$ heterotic string serving as the world sheet of another string. This by itself is not too surprising - it is well known that the dynamics on the world sheet of a string describes two-dimensional (super-) gravity, and conversely, essentially any $2 d$ gravity 
theory can be thought of as a world sheet string theory. What is nevertheless interesting here is that:

a) The $2 d$ gravity systems that arise in the target space of the $N=2$ heterotic string seem to correspond to critical string theories.

b) Critical (super-) string theories with different gauge principles can be obtained in this way, starting from closely related $(2,1)$ heterotic string vacua. In particular, small variations in the GSO projection on the world sheet of the $N=2$ string give rise in target space to the world sheet theories of twenty-six dimensional bosonic, ten dimensional type II and heterotic strings. These describe different (super-) gravities on the world sheet - the target space of the $N=2$ string - and until recently were considered quite different.

In what follows we describe the constructions that lead to the different types of strings in target space. We use the notation of section 2 .

\subsection{Bosonic strings.}

Perhaps the simplest theory of $(2,1)$ strings is one in which the spin structures of all 24 left-moving fermions $\left\{\lambda^{a}\right\}$ are identified, and one projects onto states with $(-)^{F_{L}}=1$. It is easy to write down the (world sheet) torus partition sum [11]:

$$
Z(\tau)=\frac{1}{2}\left[\left(\frac{\theta_{3}}{\eta}\right)^{12}-\left(\frac{\theta_{4}}{\eta}\right)^{12}-\left(\frac{\theta_{2}}{\eta}\right)^{12}\right] .
$$

$Z(\tau)$ is modular invariant, holomorphic in the standard fundamental domain of the modular group, $\mathcal{F}$, hence it is constant: $Z(\tau)=24$. Indeed, the spectrum of physical states consists in this case of 24 massless scalars arising from the Neveu- Schwarz (NS) sector of the internal superconformal field theory. These scalars live on the Lorentzian target space $\mathbb{R}^{1,1}$ parametrized by $x^{1}, x^{2}$ (recall that we are gauging the null current $J=\partial\left(x^{0}+x^{3}\right)$ ). We will denote $\left(x^{1} \pm x^{2}\right)$ by $x^{ \pm}$for brevity.

The vertex operators of the scalars, in the -1 picture for all three $\beta, \gamma$ systems in the problem, are:

$$
V^{a}=e^{-\phi} \lambda^{a} e^{-\bar{\phi}_{+}-\bar{\phi}_{-}} e^{i k \cdot x} ; \quad k_{+} k_{-}=0, \quad a=1, \cdots, 24 .
$$

Since the ground state energy of the Ramond $(\mathrm{R})$ sector for the left movers $\lambda^{a}$ is positive, no physical states arise from that sector. Note that we have set the momentum in the 
$x^{0}+x^{3}$ direction to zero. We could have multiplied $V^{a}$ (3.2) by $\exp \left[i q\left(x^{0}+x^{3}\right)\right]$ but since $q$ is a null vector, this would have no effect on the dynamics, leading to an equivalent representation of $V^{a}$. The gauged $U(1)$ symmetry allows one to identify the NS and $\mathrm{R}$ sectors of the right-moving $N=2$ superconformal field theory; when shifting $\mathrm{R} \rightarrow \mathrm{NS}$ one has to shift the momentum in the $\left(x^{0}+x^{3}\right)$ direction, but that is inconsequential as argued above.

The scattering amplitudes of the 24 scalars $V^{a}$ on the sphere vanish on shell, because of special properties of $N=2$ strings - see (2.4) and the subsequent discussion. Hence, the target space action is free:

$$
S_{\text {target }}=\frac{1}{\lambda^{2}} \int d^{2} x \partial_{+} V^{a} \partial_{-} V^{a} .
$$

Here $\lambda$ is the string coupling constant of the 'fundamental' $N=(2,1)$ string. Unlike more complicated string theories, where actions like (3.3) are only valid at low energy, (3.3) is exact.

The action $S_{\text {target }}$ describes free fields, but we expect the theory to contain target space gravity as well. The metric can be parametrized, after fixing diffeomorphisms, by its conformal factor, $g_{\alpha \beta}=\eta_{\alpha \beta} e^{\rho, 1}$ and in addition we expect a dilaton $\sigma$ related to the string coupling by $\left\langle e^{\sigma}\right\rangle=\lambda$. The fields $\sigma$ and $\rho$ do not describe propagating degrees of freedom - they can be gauged away. Eq. (3.3) describes the theory in a physical gauge; the covariant action (in target space) is:

$$
S_{\text {target }}=\int d^{2} x e^{-2 \sigma} \sqrt{g}\left[g^{\alpha \beta} \partial_{\alpha} V^{a} \partial_{\beta} V^{a}-\frac{1}{4} g^{\alpha \beta} \partial_{\alpha} \sigma \partial_{\beta} \sigma+R+\Lambda\right]
$$

It is natural to suggest that one should think of (3.4) as the world sheet action for a 26 dimensional bosonic string with $\left\{V^{a}\right\}$ the transverse dimensions of the string, $\sigma, \rho$ the longitudinal ones. In the target space of the $(2,1)$ string, $x^{ \pm}$is reinterpreted as the world sheet of that bosonic string. The $(2,1)$ string field space is reinterpreted as 26 dimensional spacetime. The bosonic string has infinite extent, filling the $(\rho, \sigma)$ plane.

Note that the bosonic string whose world sheet is the target space of the $(2,1)$ string has string tension $T_{B} \simeq 1 / \lambda^{2}$ (see (3.4)). Thus, string perturbation theory in the $(2,1)$ theory gives the "sigma model" $\left(\alpha^{\prime}\right)$ expansion for the bosonic string. One can ask what is

1 A different parametrization of the metric, adapted to the Kahler geometry of target space, will be explored in section 5 . 
the string coupling $\lambda_{B}$ of this bosonic string. From (3.4) it appears that the bosonic string dilaton $\Phi$ (not to be confused with the $N=(2,1)$ string dilaton, $\sigma$ ) satisfies $\Phi \simeq 1 / \lambda^{2}$. Hence a natural guess is [3]

$$
\lambda_{B} \equiv e^{\Phi} \simeq a e^{b / \lambda^{2}}
$$

with some positive constants $a, b$. When the $N=2$ string is weakly coupled, the target space bosonic string is strongly coupled.

Further insight into the structure of the theory can be obtained by studying it on a target space two-torus2. A two-dimensional torus has two complex moduli; $T$ which parametrizes the complex structure, and $U$ which measures the area and $B$ field, measured in units of $\alpha^{\prime}$. The partition sum of the $(2,1)$ string on a target space torus with moduli $T, U$ is given (perturbatively) by:

$$
\log Z_{\text {target }}(T, U, \lambda)=\sum_{h=1}^{\infty} \lambda^{2 h-2} Z_{h}(T, U),
$$

where $Z_{h}$ is the world sheet genus $h$ path integral with target space a torus with moduli $T$ and $U$. In addition one expects non-perturbative corrections in $\lambda$, which we will discuss later. The leading term in the expansion (3.6), corresponding to a world sheet torus, is obtained from the non-compact partition sum (3.1) by replacing a factor of $1 / \tau_{2}$ by a sum over momenta and windings on the 2-torus:

$$
Z_{1}(T, U)=\frac{1}{2} \cdot 24 \cdot \int_{\mathcal{F}} \frac{d^{2} \tau}{\tau_{2}^{2}} \sum_{\left(p_{L}, p_{R}\right)} q^{\frac{1}{2} p_{L}^{2}} \bar{q}^{\frac{1}{2} p_{R}^{2}}
$$

with $q=\exp [2 \pi i \tau] ;$ and $\left(T_{2} \equiv \operatorname{Im}(T) ; U_{2} \equiv \operatorname{Im}(U)\right)$ :

$$
\begin{aligned}
& p_{L}=\frac{1}{\sqrt{2 T_{2} U_{2}}}\left(n_{1}+n_{2} T+\left(m_{1}+m_{2} T\right) U\right) \\
& p_{R}=\frac{1}{\sqrt{2 T_{2} U_{2}}}\left(n_{1}+n_{2} T+\left(m_{1}+m_{2} T\right) \bar{U}\right)
\end{aligned}
$$

This integral has been computed in [12] with the result (ignoring a $T, U$ independent infrared divergent constant):

$$
Z_{1}=-24 \log \left(\sqrt{T_{2}}|\eta(T)|^{2} \sqrt{U_{2}}|\eta(U)|^{2}\right)
$$

2 One can either consider a Lorentzian torus or study the theory with positive signature. 
Comparing to (3.6) we conclude that the leading term in the target torus partition sum is:

$$
Z_{\text {target }}(T, U, \lambda)=\left(\frac{1}{\sqrt{T_{2}}|\eta(T)|^{2}}\right)^{24}\left(\frac{1}{\sqrt{U_{2}}|\eta(U)|^{2}}\right)^{24}+O\left(\lambda^{2}\right) .
$$

This looks like the partition sum of two decoupled sets of 24 scalars living on 2-tori with moduli $T, U$ respectively. These scalars are the $\left\{V^{a}\right\}$. A second set of scalars appears due to the existence of winding modes. On-shell vertex operators take the form

$$
\begin{aligned}
& V_{++}^{a}=\Xi^{a} e^{i\left(k_{+} x^{+}+\bar{k}_{+} \bar{x}^{+}\right)} \\
& V_{--}^{a}=\Xi^{a} e^{i\left(k_{-} x^{-}+\bar{k}_{-} \bar{x}^{-}\right)} \\
& V_{+-}^{a}=\Xi^{a} e^{i\left(k_{+} x^{+}+\bar{k}_{-} \bar{x}^{-}\right)} \\
& V_{-+}^{a}=\Xi^{a} e^{i\left(k_{-} x^{-}+\bar{k}_{+} \bar{x}^{+}\right)},
\end{aligned}
$$

where $\Xi^{a}=e^{-\phi} \lambda^{a} e^{-\bar{\phi}_{+}-\bar{\phi}_{-}}$. Naively, the momentum operators $V_{++}, V_{--}$only couple to deformations $T$ of the torus complex structure,

$$
T=\partial x^{+} \bar{\partial} x^{+} \quad, \quad \bar{T}=\partial x^{-} \bar{\partial} x^{-},
$$

while winding operators $V_{+-}, V_{-+}$only couple to the scale and theta parameter

$$
U=\partial x^{+} \bar{\partial} x^{-} \quad, \quad \bar{U}=\partial x^{-} \bar{\partial} x^{+} .
$$

The momentum and winding vertices $V^{a}$ correspond to, respectively, chiral-chiral and chiral-antichiral operators of the global $\mathrm{N}=(2,2)$ superconformal algebra allowed by a flat target space. Their decoupling is probably a consequence of properties of c-c and c-a rings in two-dimensional $N=2$ superconformal field theory. We leave a more detailed analysis of this issue to future work.

It is interesting to think about the structure of possible corrections to (3.10) of higher order in $\lambda$ (3.6). Suppose first that all such corrections vanished. That would imply that the scalars $\left\{V^{a}\right\}$ are non-compact, and the bosonic target space string lives in 26 noncompact dimensions. It is difficult to decide apriori from the $N=(2,1)$ string point of view whether the scalars $V^{a}$ are compact or not. We will later argue that there is (weak) evidence that they in fact live on a 24 - torus, in which case the corrections to (3.10) should turn it into:

$$
Z_{\text {target }}(T, U, \lambda)=Z_{T}(T, \lambda) Z_{U}(U, \lambda)
$$


with

$$
Z_{T}(T, \lambda)=\frac{1}{|\eta(T)|^{48}} \sum_{\left(\mathcal{P}_{R}, \mathcal{P}_{L}\right) \in \Gamma_{24,24}}\left(e^{2 \pi i T}\right)^{\frac{1}{2} \mathcal{P}_{R}^{2}}\left(e^{-2 \pi i \bar{T}}\right)^{\frac{1}{2} \mathcal{P}_{L}^{2}}
$$

where $\mathcal{P}_{R, L}$ are target-space momenta in the even self-dual lattice $\Gamma_{24,24}$, such that $Z_{T}$ is modular invariant in T. A similar structure should hold for $U$.

Since all length scales in string theory are measured in units of $\alpha^{\prime}$, and we saw that for the target space bosonic string $\alpha_{B}^{\prime} \simeq \lambda^{2}$, at weak $N=2$ coupling $\lambda \rightarrow 0$ the size of the 24-torus diverges. Thus, the fact that (3.10) appears to describe non-compact scalars $V^{a}$ may be an artifact of the weak coupling limit $\lambda \rightarrow 0$. The precise determination of the lattice $\Gamma_{24,24}$ requires the knowledge of higher orders in the perturbative expansion (3.6).

Knowledge of the perturbative series (3.6) allows one to calculate the non-perturbative corrections by using (3.6), (3.14) and modular invariance of $Z_{\text {target }}$ in $T, U$ separately. These corrections, that are due to winding modes around the 24 dimensional torus (solitons in the original $N=2$ string language), should go like $\exp \left(-c / \lambda^{2}\right)$.

One can also ask about the higher genus amplitudes of the target space string theory; one might imagine they can be analyzed by formulating the $\mathrm{N}=(2,1)$ string on a target space of the form $T^{*} \Sigma$, the cotangent space of a Riemann surface [7], or a suitable $(2,1)$ sigma-model generalization with null Killing vector.

\subsection{Type II strings.}

To construct a $(2,1)$ string theory whose target space dynamics describes a type II world sheet we modify slightly the GSO projection for the fermions $\left\{\lambda_{a}\right\}$. We split them into a set of eight and a set of sixteen. Performing separate GSO projections on the two sets we find a theory that can be alternatively described in terms of 8 scalars $y^{a}$ compactified on the $E_{8}$ torus, and 8 fermions $\lambda_{a}(a=1, \cdots, 8)$. The left-moving $N=1$ superconformal generator is

$$
G=\psi_{\mu} \partial x^{\mu}+\lambda^{a} \partial y^{a}
$$

This theory has $N=2$ superconformal symmetry and, one may hope, spacetime supersymmetry. This is indeed the case as is clear from the world sheet torus partition sum:

$$
Z(\tau)=\frac{1}{4}\left[\left(\frac{\theta_{3}}{\eta}\right)^{4}-\left(\frac{\theta_{4}}{\eta}\right)^{4}-\left(\frac{\theta_{2}}{\eta}\right)^{4}\right]\left[\left(\frac{\theta_{3}}{\eta}\right)^{8}+\left(\frac{\theta_{4}}{\eta}\right)^{8}+\left(\frac{\theta_{2}}{\eta}\right)^{8}\right] .
$$

The two factors in (3.17) correspond to the contributions of $\lambda^{a}, y^{a}$, respectively. The first factor in (3.17) vanishes due to a well known identity; the theory has the same number 
of bosons and fermions. It is easy to construct the spacetime supercharges. Denoting by $H$ the scalar obtained by bosonizing $\psi^{ \pm}$, the superpartners of $x^{ \pm}$under the left-moving superconformal algebra, and by $S_{\alpha}, S_{\bar{\alpha}}$ the dimension $1 / 2$ vertex operators creating the $s$, $c$ spinors of the $S O(8)$ associated with $\lambda_{a}$, they are:

$$
\begin{aligned}
& Q_{\alpha}=\oint d z e^{-\frac{\phi}{2}+\frac{i}{2} H} S_{\alpha} \\
& Q_{\bar{\alpha}}=\oint d z e^{-\frac{\phi}{2}-\frac{i}{2} H} S_{\bar{\alpha}}
\end{aligned}
$$

The 16 supercharges (3.18) form an $(8,8)$ supersymmetry algebra in target space:

$$
\begin{aligned}
& \left\{Q_{\alpha}, Q_{\beta}\right\}=\delta_{\alpha \beta} P^{+} \\
& \left\{Q_{\bar{\alpha}}, Q_{\bar{\beta}}\right\}=\delta_{\bar{\alpha} \bar{\beta}} P^{-} \\
& \left\{Q_{\alpha}, Q_{\bar{\beta}}\right\}=0
\end{aligned}
$$

The spectrum consists of eight massless scalars, with vertex operators:

$$
V^{a}=e^{-\phi} \lambda^{a} e^{-\bar{\phi}_{+}-\bar{\phi}_{-}} e^{i k \cdot x} ; \quad k_{+} k_{-}=0, a=1, \cdots, 8
$$

and eight right and left-moving fermions, with vertex operators:

$$
\begin{array}{ll}
\chi_{\alpha}=e^{-\frac{\phi}{2}+\frac{i}{2} H} S_{\alpha} e^{-\bar{\phi}_{+}-\bar{\phi}_{-}} e^{i k \cdot x} ; k^{+}=0 \\
\bar{\chi}_{\bar{\alpha}}=e^{-\frac{\phi}{2}-\frac{i}{2} H} S_{\bar{\alpha}} e^{-\bar{\phi}_{+}-\bar{\phi}_{-}} e^{i k \cdot x} ; k^{-}=0
\end{array}
$$

The matter content and symmetry structure is consistent with an interpretation of the target space theory as describing a type II world sheet in a physical gauge, with $V^{a}$ the eight transverse dimensions, and $\chi_{\alpha}, \bar{\chi}_{\bar{\alpha}}$ the static gauge Green-Schwarz fermions. Because one is in a physical gauge, half of the spacetime supersymmetries are realized nonlinearly. As in the bosonic construction, one can ask whether the $\left\{V^{a}\right\}$ are compact. From the form of the vertex operators for $V^{a}(3.20)$ it seems natural to guess that they are compactified on the $E_{8}$ torus. That would also mean that there is no distinction between IIA and IIB strings (the two are related by $T$ duality).

Many aspects of the discussion are similar to the bosonic case analyzed in the last subsection, and will not be repeated. In particular, the theory is free in physical gauge (just like the Green - Schwarz superstring), and one can determine the string coupling and $\alpha^{\prime}$ of the type II string that is obtained in this construction in terms of the underlying $(2,1)$ string parameters. 


\subsection{Heterotic strings 3 .}

In the discussion so far, the dynamics of the theory in the $x^{0}$ and $x^{3}$ directions was taken to be trivial. However, one should actually think of these theories as intrinsically $2+2$ dimensional. This point is driven home by the construction of heterotic strings in our framework, to which we now turn. Hořava and Witten [14] found that the $E_{8} \times E_{8}$ heterotic string could be described as an orbifold of M-theory compactified on $S^{1} / \mathbf{Z}_{2}$. This leads us to try to describe the heterotic string as a $\mathbf{Z}_{2}$ orbifold of the $(2,1)$ string. Since we are in static gauge, the M-theory world-volume is identified with three of the eleven spacetime coordinates. Therefore an orbifold of the $(2,1)$ theory should correspond to the above spacetime orbifold.

This time, we split the 24 fermions into three sets of 8 , denoted by $\left\{\lambda_{i}^{a}\right\} a=1, \cdots, 8$, $i=1, \cdots, 3$. The contribution of these fields to the left-moving supercurrent (2.5) is

$$
G=\sum_{a} \lambda_{1}^{a} \lambda_{2}^{a} \lambda_{3}^{a}
$$

corresponding to $(2.5)$ with group $[S U(2)]^{8}$. We want to preserve only half of the supersymmetries (3.18) (constructed as before from the spin fields of the first group of eight fermions, $\left.\left\{\lambda_{1}\right\}\right)$. The unbroken supercharges, say $Q_{\alpha}$, form an $(8,0)$ supersymmetry algebra on $\mathbb{R}^{1,1}$ (see (3.19)). The $\mathbf{Z}_{2}$ twist we employ is left-right asymmetric on the $(2,1)$ worldsheet, leaving the right-movers untouched, and twisting the left-moving part of the $\mathbb{R}^{2,2}$ coordinates $\left(x^{0}, x^{3}\right) \rightarrow-\left(x^{0}, x^{3}\right)$; this twist is coupled with a GSO projection on the three sets of fermions $\lambda_{i}^{a}$. This GSO projection acts as $(-1)^{F}$ separately on each of the three sets of left-moving fermions. Denote the twist field 1 of $\left(x^{0}, x^{3}\right)$ by $\sigma$; it combines with the left-moving $U(1)$ ghosts into a conformal dimension zero twist field $\Sigma=\sigma e^{\tilde{\phi} / 2}$, where $\tilde{b} \tilde{c}=\partial \tilde{\phi}$ is the number current of the $U(1)$ ghosts. Also denote by $S_{\beta}^{i}, i=2,3$ the spin fields of the fermions $\lambda_{i}^{a}$, respectively.

The spectrum of the theory includes the eight scalars $V^{a}(3.20), 8$ left-moving fermions $\bar{\chi}_{\bar{\alpha}}$ (3.21), and in addition 16 right-moving chiral bosons (described as R-R fields living at the fixed point of the $\mathbf{Z}_{2}$ twist)

$$
W_{\beta}^{i}=e^{-\frac{1}{2} \phi+\frac{i}{2} H} S_{\beta}^{i} \Sigma e^{-\frac{1}{2} \bar{\phi}_{+}-\frac{1}{2} \bar{\phi}_{-}+\frac{i}{2} \bar{H}_{2}-\frac{i}{2} \bar{H}_{1}} e^{i k \cdot x} \quad, \quad i=2,3
$$

3 For an improved treatment and further discussion, see 13.

4 Because $\left(x^{0}, x^{3}\right)$ are non-compact, there is a unique fixed point of the $\mathbf{Z}_{2}$ twist, and therefore a unique twist field. 
It is now important to include a factor of $\tilde{c}$ in the vertex operators $V^{a}, \bar{\chi}_{\bar{\alpha}}$, which we have suppressed until now. Note that the $W_{\beta}^{i}$ have non-local operator product expansions with the supercurrents $Q_{\bar{\alpha}}\left(\right.$ 3.18) and with the right-moving fermions $\chi_{\alpha}(3.21)$; hence the latter are eliminated from the spectrum. Note also that $W_{\beta}^{i}$ are inert under the unbroken $(8,0)$ supersymmetry, in agreement with their chirality.

Alternatively, one might try to replace these chiral bosons by 32 right-moving fermions. To do this, compactify $x^{3}$ on a circle whose radius is fixed by consistency; there are then two fixed points of the $\mathbf{Z}_{2}$ twist, with corresponding twist fields $\Sigma^{(s)}, s=1,2$. We can then write vertex operators

$$
F_{\beta}^{i,(s)}=e^{-\frac{1}{2} \phi+\frac{i}{2} H} S_{\beta}^{i} \Sigma^{(s)} e^{-\bar{\phi}_{+}-\bar{\phi}_{-}} e^{i k \cdot x} \quad, \quad i=2,3 \quad, \quad s=1,2 .
$$

This representation is more in the spirit of [14], as half of the right-moving current algebra arises from each fixed point. It is not clear whether one of these two representations is preferred.

The massless fields $V^{a}, \bar{\chi}_{\bar{\alpha}}$ and $W_{\beta}^{i} / F_{\beta}^{i,(s)}$ are free, as before, and apparently coincide with the field content of the heterotic string on the world sheet in the static gauge GreenSchwarz formalism. Quantization of the theory should reveal that the chiral scalars $W_{\beta}^{i}$ live on either the $E_{8} \times E_{8}$ or $\operatorname{Spin}(32) / Z_{2}$ torus, due to cancellation of gravitational anomalies in the target space of the $(2,1)$ string, but as explained previously, to see the torus more work is needed. If the $\left\{V^{a}\right\}$ are compact, the target of the target string theory will live at some point on the Narain moduli space $S O(8,24) / S O(8) \times S O(24)$.

\section{Target Space Membranes}

In the previous section we saw that the $1+1$ dimensional target space version of the $(2,1)$ string gives rise to critical (super-) strings. In this section we will demonstrate that the $1+2$ dimensional version gives (super-) membranes. Unlike strings, it is not known how to quantize membranes, and one can imagine that the $(2,1)$ string will teach us. We start with the construction of the supermembrane. 


\subsection{The eleven-dimensional supermembrane.}

In section 3.2 we have constructed a type II world sheet starting from a $(2,1)$ string with the internal left-moving superfields $\left(y^{a}, \lambda^{a}\right), a=1, \cdots, 8$, and an $N=1$ superconformal current given by (3.16). The scalars $y^{a}$ live on the $E_{8}$ torus, and the left-moving gauged $U(1)$ current was chosen to be $J=\partial x^{0}+\partial x^{3}$. This produced a theory on the $1+1$ dimensional target space parametrized by $\left(x^{1}, x^{2}\right)$, which we identified with the type II world sheet. As pointed out in [8], there is a second possible choice: gauging a left-moving $U(1)$ null current $J$ whose timelike piece lies in $\mathbb{R}^{2,2}$, and whose spatial part lies in the internal space (in this case the $E_{8}$ torus), such as:

$$
\left.J=\partial x^{0}+\partial y^{1} \quad, \quad J \mid \text { phys }\right\rangle=0 .
$$

If $x^{0}$ is initially non-compact, (4.1) effectively compactifies it, identifying the momentum in the $x^{0}$ direction with the (quantized) momentum in the $y^{1}$ direction. Therefore, the theory lives in $1+2$ non-compact dimensions, $\left(x^{1}, x^{2}, x^{3}\right)$.

The target space theory is supersymmetric. To construct the supercharges it is convenient to bosonize the left-moving fermions in a different way than before:

$$
\begin{aligned}
\psi^{1} \pm \psi^{2} & =e^{ \pm i H_{1}} \\
\psi^{3} \pm i \lambda^{2} & =e^{ \pm i H_{2}}
\end{aligned}
$$

and $\left(\lambda^{3}, \cdots, \lambda^{8}\right)$ bosonized in the standard way. Note that the fact that the $U(1)$ current (4.1) is gauged, $J \mid$ phys $\rangle=0$, is related by the superconformal algebra (3.16) to the fermionic constraint

$$
\left.\left(\psi^{0}+\lambda^{1}\right) \mid \text { phys }\right\rangle=0 .
$$

Thus, $\psi^{0}, \lambda^{1}$ decouple, and can (and will) be ignored, together with the spin $1 / 2$ bosonic ghosts that accompany (4.3).

The spacetime supersymmetry generators are constructed in the standard way out of the $S O(4)$ spinors $S_{A}, S_{\bar{A}}=e^{\frac{i}{2}\left( \pm H_{1} \pm H_{2}\right)}(A \in 2, \bar{A} \in \overline{2}$ of $S O(4))$, and the $S O(6)$ spinors $S_{\alpha}, S_{\bar{\alpha}}(\alpha \in 4, \bar{\alpha} \in \overline{4})$ constructed out of $\lambda^{3}, \cdots, \lambda^{8}$.

$$
\begin{aligned}
Q_{A \alpha} & =\oint e^{-\frac{1}{2} \phi} S_{A} S_{\alpha} \\
Q_{\bar{A} \bar{\alpha}} & =\oint e^{-\frac{1}{2} \phi} S_{\bar{A}} S_{\bar{\alpha}} .
\end{aligned}
$$


The supercharges (4.4) form as before a 16 of $S O(10)$, and decompose as $(4,2) \oplus(\overline{4}, \overline{2})$ of $S O(6) \times S O(4)$. The superalgebra is:

$$
\left\{Q_{A \alpha}, Q_{\bar{B} \bar{\beta}}\right\}=\delta_{\alpha \bar{\beta}} \gamma_{A \bar{B}}^{\mu} P_{\mu}
$$

where $P_{\mu}$ is the momentum in the $\left(x^{1}, x^{2}, x^{3}, y^{2}\right)$ directions, and $\gamma^{\mu}$ are four-dimensional $\gamma$ matrices (essentially $\sigma$ matrices). The last component of the momentum is discrete and actually vanishes for the physical on-shell states, since $\vec{p}_{y}$ belongs to an even self-dual lattice (the $E_{8}$ root lattice), and states with $\vec{p}_{y} \neq 0$ cannot satisfy $L_{0}=\bar{L}_{0}$.

There are two kinds of massless bosonic states:

$$
V^{a}=e^{-\phi} \lambda^{a} e^{-\bar{\phi}_{+}-\bar{\phi}_{-}} e^{i k \cdot x} ; a=2, \cdots, 8
$$

and

$$
A=e^{-\phi} \xi \cdot \psi e^{-\bar{\phi}_{+}-\bar{\phi}_{-}} e^{i k \cdot x}
$$

The momentum $k$ and polarization vector $\xi$ are three dimensional; the states $V^{a}$ (4.6) are seven scalars, while $A$ (4.7) is a three dimensional gauge field. In $1+2$ dimensions the gauge field has one propagating degree of freedom; it is dual to a scalar via the usual $\epsilon^{\mu \nu \rho} F_{\nu \rho}=\partial^{\mu} \phi$

There are also 8 fermions related to $V^{a}, A$ by supersymmetry (4.4). The target space theory is a theory of $2+1$ dimensional supergravity coupled to eight matter degrees of freedom. This system can be thought of as the world-volume description of an eleven-dimensional supermembrane in a physical gauge. The spectrum (4.6), (4.7) (and supersymmetry structure (4.5)) is identical to the (massless) spectrum of modes living on a Dirichlet two-brane in type IIA string theory in ten dimensions [15]. There, the analogs of $V^{a}$ are the seven collective modes for transverse fluctuations of the two-brane, while the analog of $A$ is a gauge field on the world-volume of the two-brane. The interactions in our case are somewhat different: While for D-branes the analogs of $V^{a}$ are neutral with respect to the gauge field, here there is a three-point coupling between $A$ and two $V^{a}$, as well as other couplings of the bosons (4.6), (4.7) to the fermions. Of course, in addition the Dirichlet two-brane has an infinite tower of massive excitations, and interacts in a highly nontrivial way with the modes living in the bulk of spacetime, whereas we only find massless modes and no states living away from the 'brane'. Furthermore, while in the construction of 15 the type IIA string is treated as fundamental and the Dirichlet two-brane is a soliton, we naturally regard the two-brane as fundamental and the IIA string is a limit 
of it under double dimensional reduction. The relation between the Dirichlet two-brane of the IIA theory and the membrane of eleven-dimensional supergravity has been discussed in [16, 17].

We will leave a detailed exploration of the $1+2$ dimensional dynamics of our theory to future work. It is likely that the theory describes a membrane in a $10+1$ dimensional space in a physical gauge, with $8=11-3$ physical bosonic, and 8 physical fermionic modes. Note that while the theory lives in $1+2$ non-compact directions, it should really be thought of as living in $2+2$ dimensions with one of the time coordinates compact. Due to (4.1), the on-shell physical states (4.6), (4.7) carry no momentum in the $x^{0}$ direction, but if we use the $(2,1)$ string to quantize the theory, states with $k_{0} \neq 0$ would circulate in loops. Perhaps reinterpreting the standard $1+2$ dimensional membrane as a $2+2$ dimensional theory will aid in the development of a quantum world-volume theory.

\subsection{The bosonic membrane.}

To see the importance of the $2+2$ dimensional interpretation of our theories, it is useful to construct explicitly the 'bosonic membrane' - the $1+2$ dimensional analog of the theory considered in section 3.1 .

Thus we write our $(2,1)$ string theory in terms of 24 fermions $\lambda^{a}$, with the superconformal current (2.5). We need to isolate a $U(1)$ supercurrent to gauge in this sector. To do that, choose

$$
G_{\text {int }}=\lambda^{1} \lambda^{2} \lambda^{3}+\frac{1}{6} f_{a b c} \lambda^{a} \lambda^{b} \lambda^{c},
$$

with $a, b, c=4, \ldots, 24$ and $f_{a b c}$ the structure constants of a 21-dimensional Lie algebra. The gauged $U(1)$ supercurrent is then taken as

$$
\begin{array}{ll}
J(z)=\partial x^{0}+\lambda^{2} \lambda^{3} \quad ; & J \mid \text { phys }\rangle=0 \\
\psi(z)=\psi^{0}+\lambda^{1} ; & \psi \mid \text { phys }\rangle=0 .
\end{array}
$$

Equation (4.9) relates $k_{0}$ to the $\lambda^{2}, \lambda^{3}$ fermion number. The physical states consist of 21 massless scalars

$$
V^{a}=e^{-\phi} \lambda^{a} e^{-\bar{\phi}_{+}-\bar{\phi}_{-}} e^{i k \cdot x} \quad, \quad a=4, \ldots, 24
$$

in addition one finds

$$
\begin{aligned}
& T^{+}=e^{-\phi}\left(\lambda^{2}+i \lambda^{3}\right) e^{-\bar{\phi}_{+}-\bar{\phi}_{-}} e^{i x^{0}} e^{i k \cdot x} \\
& T^{-}=e^{-\phi}\left(\lambda^{2}-i \lambda^{3}\right) e^{-\bar{\phi}_{+}-\bar{\phi}_{-}} e^{-i x^{0}} e^{i k \cdot x}
\end{aligned}
$$


these states are scalars that are massless in four dimensions, but in $1+2$ dimensions they are tachyonic with $m^{2}=-1$ :

$$
E^{2}=\left(k_{1}\right)^{2}=k_{2}^{2}+k_{3}^{2}-1
$$

because they carry quantized momentum in the timelike $x^{0}$ direction. Finally, we have a three-dimensional gauge field

$$
A=e^{-\phi} \xi \cdot \psi e^{-\bar{\phi}_{+}-\bar{\phi}_{-}} e^{i k \cdot x}
$$

with $\xi \cdot k=0, \xi \approx \xi+\epsilon k$ as before.

The fact that $T^{ \pm}$are tachyons in $1+2$ dimensions does not lead to infrared divergences in the one-loop amplitude of the $(2,1)$ string, as one would ordinarily expect in a field theory with tachyons. One simply has to revert to the $2+2$ dimensional view of the theory. This seems to indicate that we should think of the theory not as a membrane in $26+1$ dimensions, but rather as a theory of a membrane with two world-volume time coordinates, one of which is compact, living in $26+2$ dimensions. Presumably the same holds for the supermembrane, which should then be thought of as having a worldsheet of signature $2+2$ and living in $10+2$ dimensional spacetime.

\section{General Backgrounds}

So far we have concentrated on the string in a flat background; however, the topological perturbation theory of the target string or membrane requires us to consider the $\mathrm{N}=(2,1)$ theory on a curved target space. To that end, let us review the beta function equations of $(2,1)$ sigma models [18, 19].

The $(2,0)$ superspace description of $\mathrm{N}=(2,1)$ heterotic strings involves real scalar chiral superfields $\mathcal{X}^{\mu}, \mu=0,1,2,3$ with signature $(-,-,+,+)$. Pairing these into light-front coordinates

$$
\begin{array}{ll}
X^{1}=\mathcal{X}^{0}+\mathcal{X}^{3} & X^{\overline{1}}=\mathcal{X}^{0}-\mathcal{X}^{3} \\
X^{2}=\mathcal{X}^{1}+\mathcal{X}^{2} & X^{\overline{2}}=\mathcal{X}^{1}-\mathcal{X}^{2},
\end{array}
$$

the needed $(2,0)$ superfields are

$$
X^{i}\left(z ; \bar{z}, \bar{\theta}_{ \pm}\right)=x^{i}+\bar{\theta}_{+} \bar{\psi}^{i}+\cdots \quad, \quad X^{\bar{i}}\left(z ; \bar{z}, \bar{\theta}_{ \pm}\right)=x^{\bar{i}}+\bar{\theta}_{-} \bar{\psi}^{\bar{i}}+\cdots
$$


satisfying $\bar{D}_{-} X^{i}=\bar{D}_{+} X^{\bar{i}}=0$. In addition, there are 28 left-moving fermionic superfields $\Lambda^{a}\left(z ; \bar{z}, \bar{\theta}_{ \pm}\right)=\lambda^{a}+\cdots$ satisfying $\bar{D}_{-} \Lambda^{a}=W^{a}$ for some superfield with $\bar{D}_{-} W^{a}=0$. The action is

$$
S=-\frac{1}{2} \int d^{2} z d^{2} \bar{\theta}\left[i\left(K_{i}(X, \bar{X}) \partial X^{i}-K_{\bar{i}}(X, \bar{X}) \partial X^{\bar{i}}\right)+V_{a^{*} b}(X, \bar{X})\left(\Lambda^{a}\right)^{*} \Lambda^{b}\right]
$$

Consistency requires (a) that the target space parametrized by $X, \bar{X}$ have signature $4+0$ or $2+2$ (we will concentrate on the latter); (b) that there exist a holomorphic supercurrent $G=e_{\mu a}(\mathcal{X}) \Lambda^{a} \partial \mathcal{X}^{\mu}+f_{a b c}(\mathcal{X}) \Lambda^{a} \Lambda^{b} \Lambda^{c} ;$ and (c) that we gauge an anomaly-free, left-moving current $\mathcal{J}=\mathcal{V}_{a}(\mathcal{X}) \Lambda^{a}$ and its holomorphic superpartner.

In the free theory (flat spacetime), the background is

$$
K_{i}=\delta_{i, \bar{i}} X^{\bar{i}}, \quad K_{\bar{i}}=\delta_{\bar{i}, i} X^{i}, \quad V_{a b}=\text { const. } .
$$

The N=1 left-moving supercurrent is obtained by splitting off four of the twenty-eight $\Lambda$ 's, call them $\Psi^{i}, \Psi^{\bar{i}}$, and pairing them with the $X^{i}, X^{\bar{i}}$. Then $e_{\mu a}=\delta_{\mu a}$, and a solution for $f_{a b c}$ may be determined by putting the remaining $\Lambda^{a}$ in the adjoint of some semisimple group of dimension 24 [11] (for which the $f_{a b c}$ are the structure constants). Note that (5.4) looks like the embedding of a $(2+2)$ - brane into a $26+2$ dimensional spacetime.

The semi-Kahler potential $K_{\mu}$ of (5.3) determines the metric and torsion in holomorphic coordinates:

$$
g_{i \bar{j}}+b_{i \bar{j}}=K_{i, \bar{j}} \quad g_{i j}=b_{i j}=0 .
$$

It proves convenient to use the connection with torsion $\Gamma_{\nu \lambda}^{\mu}=\gamma_{\nu \lambda}^{\mu}-\frac{1}{2} T_{\nu \lambda}^{\mu}(\gamma$ is the Christoffel connection). The torsion is determined as

$$
T_{i j \bar{k}}=\partial_{\bar{k}}\left(K_{i, j}-K_{j, i}\right) \quad T_{i j k}=0,
$$

and the one-loop beta function equations $R_{\mu \nu}^{(-)}=D_{\mu} D_{\nu} \phi$ boil down to

$$
\begin{aligned}
V_{i}=T_{k i}^{k} & =\frac{1}{2} \partial_{i} \phi^{*} \\
V_{\bar{i}}=T_{\bar{k} \bar{i}}^{\bar{k}} & =\frac{1}{2} \partial_{\bar{i}} \phi \\
\exp \left[\phi+\phi^{*}\right] & =\operatorname{det}[g] \\
g^{i \bar{j}} D_{i} \partial_{\bar{j}} \phi & =g^{\bar{i} j} D_{\bar{i}} \partial_{j} \phi^{*}=0 .
\end{aligned}
$$


Here $\phi+\phi^{*}$ is the dilaton of the $(2,1)$ string, and $\phi-\phi^{*}$ is the theta parameter of its abelian world sheet gauge theory. Note that the equations are invariant under the gauge transformation

$$
\delta K_{i}=\partial_{i} \chi \quad, \quad \delta K_{\bar{i}}=-\partial_{\bar{i}} \chi ;
$$

hence, counting degrees of freedom in the gravity sector, we should have four - two from the dilaton and theta fields, and two from $K_{\mu}$ (four minus one gauge invariance and one gauge condition). The worldsheet fields $\Lambda$ give rise to self-dual Yang-Mills in the $2+2$ target space, parametrized by a single adjoint scalar field (e.g. 24 scalars for the 'bosonic string' analog). At one loop, anomaly cancellation requires $d T=\operatorname{tr}\left[R^{2}-F^{2}\right]$. The YangMills field strength $F$ enters the beta function equations first at two loops; one finds curvature-squared corrections to (5.7) [18]:

$$
R_{\mu \nu}^{(-)} \longrightarrow R_{\mu \nu}^{(-)}+\frac{\alpha^{\prime}}{4}\left[R_{\mu}^{(+) \rho \sigma \tau} R_{\rho \sigma \tau \nu}^{(+)}-F_{\mu \rho} F_{\nu}{ }^{\rho}\right],
$$

where the superscripts denote the curvature of the connection $\Gamma^{( \pm)}=\gamma \pm T$. If we want to regard the field space of this theory as the target space of a $2+2$ world volume, our off-shell counting of target space dimension agrees with the expectation generated by the analysis of sections three and four - $\operatorname{dim}(G)$ (e.g. twenty-four) transverse coordinates together with $2+2$ longitudinal coordinates from the gravity sector. Note that $d T-\operatorname{tr}\left[R^{2}-F^{2}\right]=0$ looks a bit like a Virasoro constraint; indeed, it comes from a reparametrization anomaly in the sigma-model field space.

We have not analyzed the conditions for a left-moving null current algebra and supersymmetry. In the case of reduction to $1+1$ dimensions the appearance of a current algebra should amount to the requirement that the $2+2$ geometry admit a covariantly constant null Killing vector. Target space supersymmetry requires the $(2,1)$ sigma model carry an $\mathrm{N}=(2,2)$ global supersymmetry, which further constrains the semi-Kahler potential; the torsion connection $\Gamma$ is embedded in the gauge group. The details of the sigma model geometry may help uncover the $10+2$ supergravity theory hinted at by our work.

\section{Comments and Speculations}

Our results open a number of intriguing avenues of investigation. Among the potential applications of our work are: 
i) As an indication of substructure to world-volumes. This was one of the main motivations of [3]. Here, one finds a sequence: World sheet $\hookrightarrow$ self-dual four-space $\hookrightarrow$ spacetime. Since different string theories are related on the first level by orbifold and deformation of the null current, and on the last level by strong/weak coupling duality, one might wonder whether there is another link: $(2,1)$ world sheet $\leftrightarrow$ spacetime.

ii) As an indication of how to construct the quantum theory of membranes directly as a quantum field theory in $2+2$ dimensions. One imagines using the $(2,1)$ string as a sort of scaffolding to construct such a theory, to be removed upon completion.

iii) As a novel construction of soliton strings/membranes describing critical string/Mtheory compactified down to two or three dimensions.

Let us comment on these and a few related topics.

\subsection{The $N=(2,0)$ string.}

The $(2,0)$ string is a theory obtained by combining a right moving $N=2$ string with a left moving bosonic string (see e.g. [8]). The simplest vacua correspond to compactifications of the left movers on one of the 24 Niemeier tori. In his original paper, Green pointed out that the theory obtained by compactifying on the Leech torus is a critical bosonic string. It would be interesting to understand these theories in general. Like the $(2,1)$ strings discussed above, they only have massless excitations, and when the target space is $1+1$ dimensional, these are free.

There are two differences:

a) Even in the simplest constructions mentioned above, the $(2,0)$ string generically gives rise to non-critical string theories in target space. Indeed, the spectrum of the theory on a particular Niemeier lattice includes the 24 oscillator modes $\partial y^{a}$, and in addition momentum states $\exp [i \vec{p} \cdot \vec{y}]$ with $p^{2}=2$. These massless states form the adjoint representation of a rank 24 group $G$ which depends on the lattice (for the Leech lattice the group is $U(1)^{24}$ but in general it is more complicated).

b) The decoupling of "momentum" and "winding" strings, described for the $(2,1)$ case in section 3 (see $(\sqrt{3.10}))$ does not occur here. The analog of the expression for $Z_{1}$ (3.7) in this case is:

$$
Z_{1}(T, U)=\frac{1}{2} \int_{\mathcal{F}} \frac{d^{2} \tau}{\tau_{2}^{2}}\left(J(\tau)+C_{0}\right) \sum_{\left(p_{L}, p_{R}\right)} q^{\frac{1}{2} p_{L}^{2}} \bar{q}^{\frac{1}{2} p_{R}^{2}}
$$


where $J(\tau)=1 / q+\cdots$ is the unique meromorphic modular invariant function with the specified behavior as $q \rightarrow 0$ and no constant term in the expansion in powers of $q$. $C_{0}$ is the number of massless states, equal to the dimension of the group $G$ corresponding to the particular Niemeier lattice.

This integral has been computed in [20], with the result:

$$
Z_{1}(T, U)=-C_{0} \log \left(\sqrt{T_{2}}|\eta(T)|^{2} \sqrt{U_{2}}|\eta(U)|^{2}\right)-\log |J(T)-J(U)|^{2} .
$$

The first term on the right hand side is just what one would expect for a pair of non-critical strings living on the $T$ and $U$ tori as for the $(2,1)$ string discussed in section 3 . The second term on the r.h.s. means (comparing (6.2) to (3.9)) that the decoupling between $T$ and $U$ that took place there does not occur for the $(2,0)$ string, probably because of the lack of an $N=2$ superconformal structure in the left moving sector. It would be interesting to understand these theories better.

\subsection{Strong/weak coupling duality.}

The S-duality group of the $(2,1)$ string is the T-duality group of its target string theory; thus $(2,1)$ strings may serve as a useful laboratory for probing the structure of Sduality in string theory, given that we understand T-duality rather well. We also have yet another context in which to investigate target space topology change, given that eventually one will have to sum over the topology of the target space. This is certainly true in the $1+1$ dimensional reduction, and likely to be true in the $2+1$ reduction as well. There is also the possibility, given that we are on the road to an explicit description of quantum membranes, that we will understand the rules for constructing orbifolds of M-theory (a hint of which we saw in section 3.3), the T-duality structure of M-theory, and so on.

Hull [1] has pointed out that a twelve-dimensional origin of string theory would explain the $S L(2, \mathbf{Z})$ S-duality of type IIB strings in ten dimensions as the T-duality symmetry of a reduction from twelve to ten dimensions on a two-torus. One would like to see how that arises in our framework.

Different choices of GSO projection of the $(2,1)$ string give rise to different critical (super-) string theories. Yet we know another way that these theories are connected: Strong-weak coupling duality. Could it be that the S-duality group of the various string and M-theories is related to the discrete symmetry group of $(2,1)$ strings? This group includes the group of GSO projections. One would also need to include the discrete choice

of null current orientation in such a construction. This exciting possibility would close the chain of embeddings of parameter space into field space that makes spacetime from $2+2$ world volume from $(2,1)$ string worldsheet. 


\subsection{Analogies with soliton strings.}

There is another possible interpretation of our construction based on its similarity to that of solitonic strings and other $p$-branes in higher-dimensional string theory [15], [21]. Indeed, the target geometry we described was that of a $p$-brane world sheet stretched across all of $p+1$ dimensional spacetime, for $p=1,2$. The solution breaks half of the supersymmetries, which arise in a Green-Schwarz type formalism. The tension of the target brane is related to the string coupling of the $(2,1)$ string, and when the target space is two dimensional, the coupling of the target string goes to infinity as the coupling of the $(2,1)$ string goes to zero.

In higher dimensional string theories, soliton string constructions often point to strongweak coupling dual pairs. One may ask whether it is possible that this is also the case here. Specifically, could it be that a strongly coupled ten dimensional heterotic or type II string compactified on an 8 torus is equivalent to a weakly coupled two dimensional $(2,1)$ string? At first sight this seems ludicrous: a heterotic or type II string theory has, even in a two dimensional spacetime, an infinite tower of states (with exponential density of states at large mass), while the $(2,1)$ string describes a small collection of massless two dimensional field theoretic degrees of freedom with no massive states at all.

One may ask, what happened to the exponential density of BPS states of the critical string? By definition, they are all charged under some U(1) gauge group, which confines in two dimensions; there are no BPS states in the physical asymptotic spectrum! What remains are the 'moduli fields' and gravity. Gravity merely dresses the renormalization group flows of various operators. The moduli fields parametrize a nonlinear sigma model; they are disorderd in two dimensions; the theory does not have a manifold of ground states. The moduli sigma model is infrared free (at weak coupling) - the theory flows towards $8 \times 8$ or $8 \times 24$ free fields coupled to dilaton gravity, i.e. a noncritical string. This seems in disagreement with the massless spectrum of the $(2,1)$ string, which contains 8 right moving and either 8 or 24 left moving scalars. However, we saw in section 3 that when the $(2,1)$ string is weakly coupled, the target theory is very strongly coupled; it is not completely clear what the sigma model dynamics is in this situation. In fact, examples are known in $\mathrm{N}=1$ supersymmetric Yang-Mills in four dimensions [22] whereby a naively irrelevant flow at weak coupling is nevertheless relevant at strong coupling and drives the theory to a nontrivial fixed point. Could that be happening here? It seems suggestive that the counting of moduli fields of toroidally compactified superstrings agrees with that of mesons made from the target fields of the $(2,1)$ string. 
A similar discussion would lead one to conclude that the bosonic string that is well known to be perturbatively unstable at weak coupling, is in fact stable at strong coupling (at least when compactified to $1+1$ spacetime dimensions), and is equivalent to a version of the $(2,1)$ string, described in section 3 .

If the above discussion is valid, it implies that strongly coupled $2 d$ strings have vastly fewer degrees of freedom than one would deduce perturbatively, an idea that has been suggested in various contexts in the past.

\subsection{New principles.}

The reduction of four-dimensional self-duality to yield both string and membrane world-volume theories suggests that one might elevate self-duality to a principle underlying unification, superseding the principle of two-dimensional conformal invariance which has been so fruitful in developing our understanding of strings. To this end, we need to generalize our treatment above, which took place in a physical gauge where spacetime Lorentz invariance and half the supersymmetries are nonlinearly realized. Similar issues have arisen in the effort to find an acceptable covariant quantization of the Green-Schwarz string formalism; there, the use of twistor methods [23] has helped elucidate the geometrical structure. Roughly, the twistor variables covariantize the breaking of $S O(9,1)$ Lorentz symmetry down to the little group $S O(1,1) \times S O(8)$, and at the same time bring in fascinating connections to Hopf fibrations, Jordan algebras, octonions, lightlike integrability, etc. A natural starting point might be to generalize these methods to the conformal group $S O(10,2)$, which also has a twistorial construction. The null gauging that appears in the $\mathrm{N}=(2,1)$ string might have an interpretation in terms of group contractions of this larger (super-) symmetry. Note, in particular, that central charges arise in this way [24].

We do not necessarily mean to suggest that the only way to obtain a quantum theory of membranes is to construct a string theory whose target space is the membrane worldvolume. Rather, one might interpret the $(2,1)$ heterotic string as a guide toward the construction of an appropriate world-volume quantum field theory. It is not out of the question that one could quantize directly the reduced version of self-dual gravity coupled to self-dual matter that appears as the effective target dynamics of the $(2,1)$ string. First of all, the fluctuations of the geometry are restricted to be self-dual even off-shell; second, the null gauging of the $(2,1)$ string leads to further restriction on the target fluctuations. These may be enough to render the theory renormalizable. Note that the usual string effective Lagrangian $e^{-2 \phi} \sqrt{g}[R+\cdots]$ leads to improved power counting if we use $g_{i \bar{j}}=\partial_{(i} K_{\bar{j})}$ and 
treat $K$ as the fundamental dynamical field. Constraints on matter content (i.e. $10+2$ or $26+2$ dimensions of spacetime) should arise from quantum anomalies in self-dual gravity Ward identities in $2+2$ dimensions.

If there is secretly a second timelike coordinate that only appears in off-shell dynamics, what are its consequences? Could there be novel mechanisms for solving some of the longstanding problems of unification? Supersymmetry breaking, the cosmological constant, and other hierarchy problems come to mind; as well as the horizon problem in cosmology and the Hawking paradox. After all, the causal structure of spacetime is subtly different in this theory; and the second, off-shell time direction could allow communication between vastly different energy and time scales of the principal time coordinate via fluctuations travelling along the second time. Another fascinating feature of the $(2,1)$ string is the joint appearance of two apparently decoupled string theories living in mirror target spaces; can they be coupled (perturbatively or nonperturbatively), as they are in $(2,0)$ strings? What would this mean?

\subsection{Related ideas.}

The constructions in this paper touch on a number of ideas about underlying structure in string theory that have been proposed over the years:

a) The complexification of the world sheet and spacetime [25]. These have been considered before, in studies of high-energy/temperature behavior of strings.

b) Worldsheet - target space duality. Giveon et.al. [26] noticed that a string compactified on a target torus exhibits a similarity between the dependence on the Narain moduli of the target space and the world sheet period matrix.

c) The possibility of a hidden relation between bosonic and fermionic strings. Freund [27] was the first to suggest that one might be able to obtain the fermionic string from the bosonic string.

d) That the 'number of degrees of freedom' in string theory (whatever that means) is vastly reduced at short distances [28], or high temperatures [29] has been suggested by several independent approaches; there are also indications of two-dimensional structure in the asymptotic spectrum [30]. These ideas resonate strongly with the interpretation of the $(2,1)$ string as a strong-coupling dual of critical string theories, given its two-dimensional field-theoretic density of states.

e) We have seen in sections 3 and 4 (see also [8]) that the $(2,1)$ string secretly does live in $2+2$ dimensions, in some instances with one of the time directions compactified. 
This leads to the possible emergence of hyperbolic algebras as a hidden symmetry of string/membrane theory; for instance, with one space and one time direction of the $2+2$ spacetime compactified, the right-moving dynamics involves $\Gamma_{1,1}$, while that of the left movers takes place in $\Gamma_{9,1}$. Structures of this sort have been considered in [31,32]. Note that there is a unique null vector in such lattices; this may lead to a common treatment of strings and membranes within our framework.

f) More than 10 or 11 dimensions. Attempts have been made before to go beyond the barrier of eleven as the maximal dimension of supergravity [33]. Hull recently presented indirect evidence for the existence of such a theory on the basis of lowerdimensional vacua of string/M-theory [1]; further evidence was presented in [34]35].

Acknowledgements: It is a pleasure to thank M. Douglas, P.G.O. Freund, J. Harvey, and M. O'Loughlin for discussions. 


\section{References}

[1] C. Hull, hep-th/9512181.

[2] E. Bergshoeff, E. Sezgin and P. Townsend, Ann. Phys. 185 (1988) 330.

[3] M. Green, Nucl. Phys. B293 (1987) 593.

[4] N. Marcus, talk at the Rome String Theory Workshop (1992); hep-th/9211059.

[5] M. Ademollo et.al., Phys. Lett. 62B (1976) 105, Nucl. Phys. B111 (1976) 77; E. S. Fradkin and A. A. Tseytlin, Phys. Lett. 106B (1981) 63, Phys. Lett. 162B (1985) 295; A. D'Adda and F. Lizzi, Phys. Lett. 191B (1987) 85; S. Mathur and S. Mukhi, Phys. Rev. D36 (1987) 465; Nucl. Phys. B302 (1988) 130.

[6] H. Ooguri and C. Vafa, Mod. Phys. Lett. A5 (1990) 1389.

[7] H. Ooguri and C. Vafa, Nucl. Phys. B361 (1991) 469.

[8] H. Ooguri and C. Vafa, Nucl. Phys. B367 (1991) 83.

[9] E. Witten, hep-th/9410052; A. Galperin, E. Sokatchev, hep-th/9504124.

[10] D. Friedan, E. Martinec and S. Shenker, Nucl. Phys. B271 (1986) 93.

[11] D. Pierce, hep-th/9601125.

[12] L. Dixon, V. Kaplunovsky, and J. Louis, Nucl. Phys. B355 (1991) 649; see also A. Font, L. Ibanez, D. Lüst, and F. Quevedo, Phys. Lett. 245B (1990) 401; S. Ferrara, N. Magnoli, T. Taylor, and G. Veneziano, Phys. Lett. 245B (1990) 409.

[13] D. Kutasov, E. Martinec, and M. O'Loughlin, hep-th/9603116.

[14] P. Hořava and E. Witten, hep-th/9510209.

[15] J. Polchinski, hep-th/9510017, Phys. Rev. Lett. 75 (1995) 4724.

[16] P. Townsend, hep-th/9512062.

[17] C. Schmidhuber, hep-th/9601003.

[18] C.M. Hull, Phys. Lett. 178B (1986) 357.

[19] G. Bonneau and G. Valent, Class. Quant. Grav. 11 (1994) 1133; hep-th/9401003.

[20] G. Lopes Cardoso, D. Lüst, and T. Mohaupt, Nucl. Phys. B450 (1995) 115; hepth/9412209.

[21] J. Harvey and A. Strominger, hep-th/9504047, Nucl. Phys. B449 (1995) 535; A. Sen, hep-th/9504027, Nucl. Phys. B450 (1995) 103; D. Kutasov, hep-th/9512145.

[22] D. Kutasov, A. Schwimmer and N. Seiberg, hep-th/9510222.

[23] F. Delduc, A. Galperin, P. Howe, and E. Sokatchev, Phys. Rev. D47 (1993) 578; hep-th/9207050.

[24] J. Lukierski and L. Rytel, J. Phys. A15 (1982) L215.

[25] E. Witten, Phys. Rev. Lett. 61 (1988) 670.

[26] A. Giveon, N. Malkin and E. Rabinovici, Phys. Lett. 220B (1989) 551.

[27] P. G. O. Freund, Phys. Lett. 151B (1985) 387. See also A. Casher, F. Englert, H. Nicolai, and A. Taormina, Phys. Lett. 162B (1985) 121; F. Englert, H. Nicolai, and A. Schellekens, Nucl. Phys. B274 (1986) 315. 
[28] I. Klebanov and L. Susskind, Nucl. Phys. B309 (1988) 175.

[29] J. Atick and E. Witten, Nucl. Phys. B310 (1988) 291.

[30] D. Kutasov and N. Seiberg, Nucl. Phys. B358 (1991) 600.

[31] G. Moore, hep-th/9305139.

[32] J. Harvey and G. Moore, hep-th/9510182.

[33] L. Castellani, P. Fre, F. Giani, K. Pilch, and P. van Nieuwenhuizen, Phys. Rev. D26 (1982) 1481; M. Wang, Supergravity in twelve dimensions, in the Proceedings of the Marcel Grossmann Meeting, Rome 1985, p. 1459; P. G. O. Freund, Introduction to Supersymmetry, Cambridge Press, 1986, ch. 26 (!); M. Blencowe and M. Duff, Nucl. Phys. B310 (1988) 387.

[34] C. Vafa, hep-th/9602022; D. Morrison and C. Vafa, hep-th/9602114.

[35] A. A. Tseytlin, hep-th/9602064. 